\title{
Hook Wire Placement Facilitates Laparoscopic Excision of Endophytic Renal Tumor in Partial Nephrectomy
}

\author{
Qusay Mandoorah, MD, Francois Rozet, MD, Fabio Muttin, MD, Eric Barret, MD, \\ Rafael Sanchez-Salas, MD, and Xavier Cathelineau, MD
}

\begin{abstract}
Introduction: Endophytic renal tumors that are completely intraparenchymal pose several challenges to surgeons, including in intraoperative tumor identification. Image-guided hook wires, which are now used in surgery, particularly in spinal surgery, thoracoscopic surgery, and breast surgery, allow for the precise localization of tumor sites. The hook wire facilitated the localization of the lesion and avoided cutting into the lesion directly.

Case Presentation: A 55-year-old woman was referred to our hospital due to an incidentally discovered 16-mm intracortical right renal mass in the anterior medial position. A renal biopsy was performed, which confirmed renal cell carcinoma. A hook wire was placed in the tumor by an interventional radiologist under CT guidance. This was done before performing the partial nephrectomy on the same day. The hook wire was found intraoperatively, and the renal artery was clamped. The renal capsule was resected using scissors under warm ischemia (25 minutes). Histopathology confirmed clear-cell renal carcinoma with negative surgical margins, Classification TNM 2017: pT1a Nx.
\end{abstract}

Conclusion: Use of a hook wire is an alternative method for localizing endophytic lesions in partial nephrectomy.

Keywords: partial nephrectomy, hook wire, endophytic renal tumor

\section{Introduction}

$\mathbf{E}$ NDOPHYTIC RENAL TUMORS that are completely intraparenchymal pose several challenges to surgeons, including an intraoperative tumor identification. ${ }^{1}$ Imageguided hook wires, which are now used in surgery, particularly in spinal surgery, thoracoscopic surgery, and breast surgery, allow for the precise localization of tumor sites. $^{2,3}$ We report the case of a woman undergoing laparoscopic partial nephrectomy in which a hook wire was used. The hook wire facilitated the localization of the lesion and avoided cutting into the lesion directly. We found one case report involving embolization coil placement. ${ }^{4}$

\section{Case Presentation}

A 55-year-old woman was referred to our hospital due to an incidentally discovered $16-\mathrm{mm}$ intracortical right renal mass in the anterior medial position (Fig. 1). A renal biopsy was performed, which confirmed renal cell carcinoma.
A hook wire was placed in the tumor by an interventional radiologist under CT guidance. This was done before performing the partial nephrectomy on the same day using the technique used for nonpalpable breast lesions to facilitate intraoperative localization of the tumor (Figs. 2 and 3).

The patient was placed in a modified left lateral decubitus position. Pneumoperitoneum was established and the trocars were placed. The bowel was mobilized medially and the plane between the anterior Gerota's fascia and the posterior mesocolon was developed. The kidney was mobilized within Gerota's fascia. The hook wire was found, and the renal artery was clamped by the bulldog. The renal capsule was resected using scissors under warm ischemia (25 minutes) (Fig. 4).

Hemostasis was achieved using a combination of cautery, hemostatic agents, and suturing (using sutures preloaded with clips to secure a suture line allows for lateral compression, perpendicular to the renal capsule).

Histopathology confirmed clear-cell renal carcinoma with negative surgical margins, Classification TNM 2017: pT1a Nx.

Department of Urology, Institut Mutualiste Montsouris, Paris, France.

(C) Qusay Mandoorah et al. 2018; Published by Mary Ann Liebert, Inc. This Open Access article is distributed under the terms of the Creative Commons License (http://creativecommons.org/licenses/by/4.0), which permits unrestricted use, distribution, and reproduction in any medium, provided the original work is properly cited. 


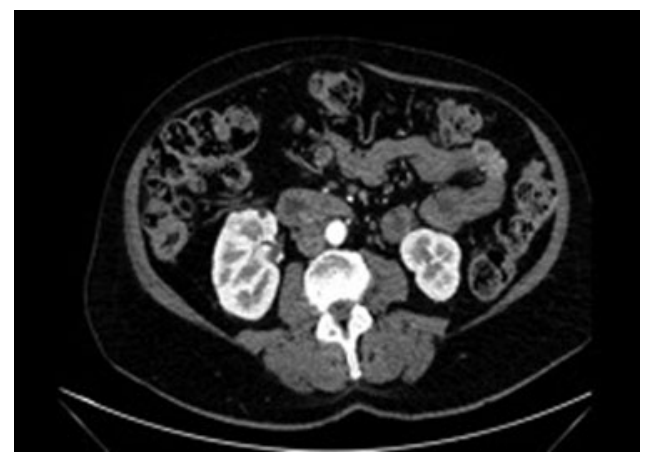

FIG. 1. Computed tomographic scan views of the right renal mass.

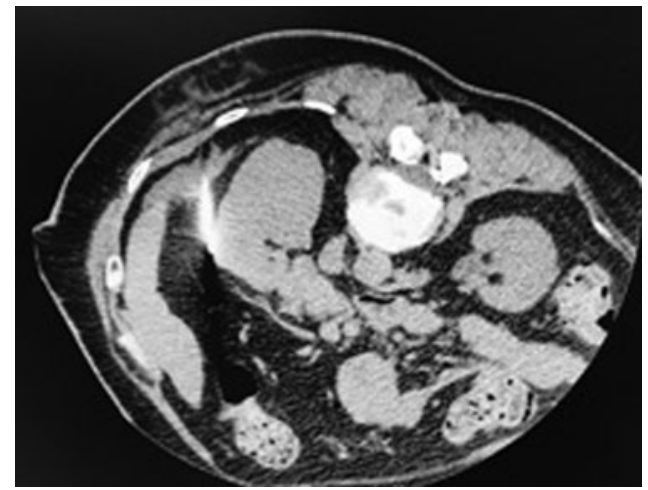

FIG. 2. Computed tomographic scan views after the placement of the hook wire.

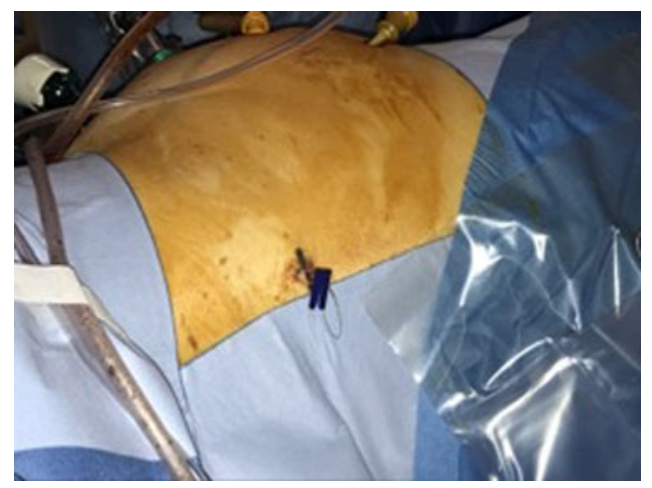

FIG. 3. Placement of the hook wire extracorporeal.

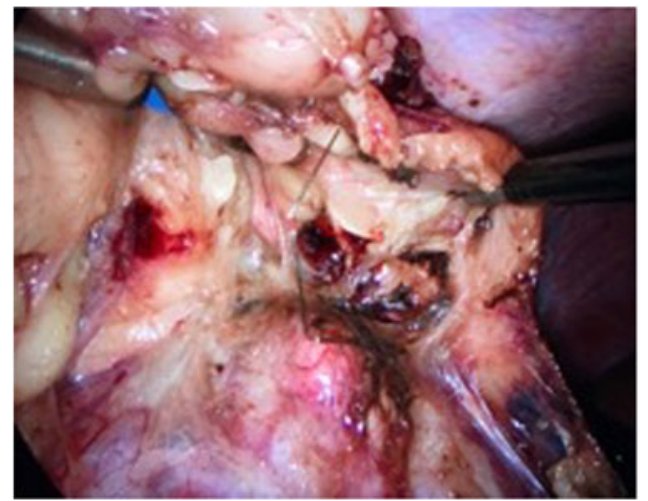

FIG. 4. Intraoperative view; the hook wire can be observed.

\section{Discussion}

Laparoscopic partial nephrectomy for endophytic renal masses is technically challenging, as the surgeon does not have intraoperative gross observation of the renal mass and recognition of the mass extension. Image-guided hook wires, which are now used in surgery, particularly in spinal surgery, thoracoscopic surgery, and breast surgery, allow for the precise localization of tumor sites. The advantages of using this technique are intraoperatively easy to identify the needle and short localization procedure time. However, it also has disadvantages including hook wire dislodgement that could occur during patient transport and positioning or surgeon manipulation of the kidney.

Intraoperative ultrasonography is widely utilized for localization. It improves the identification of the renal mass location and facilitates complete tumor excision with negative surgical margins. Real-time three-dimensional (3D) image guidance system by Tilepro ${ }^{\mathrm{TM}}$ is reportedly also used. This system (renal tumor and vascular 3D image navigation shown on the surgeon console view through Tilepro) facilitates tumor and vascular identification, precise dissection of both the tumor and the pedicle, and selective clamp techniques. Another technique that facilitates tumor identification is near-infrared fluorescence imaging using indocyanine green dye. ${ }^{1}$ In the absence of these techniques, the hook wire technique is an alternative method for tumor localization and can be combined with intraoperative ultrasonography.

\section{Conclusion}

Use of a hook wire is an alternative method for localizing endophytic lesions in partial nephrectomy.

\section{Acknowledgment}

This research did not receive any specific grant from funding agencies in the public, commercial, or nonprofit sectors.

\section{Ethical Statement}

Consent was provided by the patient.

\section{Disclosure Statement}

No competing financial interests exist.

\section{References}

1. Komninos C, Shin TY, Tuliao P, Kim DK, Han WK, Chung BH, Choi YD, Rha KH. Robotic partial nephrectomy for completely endophytic renal tumors: Complications and functional and oncologic outcomes during a 4-year median period of follow-up. Urology 2014;84:1367-1373.

2. Agrawal V, Sharma A, Wu G. Preoperative fiducial coil placement facilitates robot-assisted laparoscopic excision of retroperitoneal small solitary metastasis of kidney cancer. Urology 2014;84:e21-2e22.

3. Zhao G, Sun L, Geng G, et al. Semi-rigid single hook localization the best method for localizing ground glass opacities during video-assisted thoracoscopic surgery: Reaerated swine lung experimental and primary clinical results. J Thorac Dis 2017;9:5161-5170. 
4. Reeves JJ, Forauer A, Seigne JD, Hyams ES. Image-guided embolization coil placement for identification of an endophytic, isoechoic renal mass during robotic partial nephrectomy. J Endourol Case Rep 2015;1:59-61.

Address correspondence to: Qusay Mandoorah, MD Department of Urology Institut Mutualiste Montsouris 42 Boulevard Jourdan Paris 75014

France

E-mail: mandoorah104@gmail.com
Cite this article as: Mandoorah Q, Rozet F, Muttin F, Barret E, Sanchez-Salas R, Cathelineau X (2018)

Hook wire placement facilitates laparoscopic excision of endophytic renal tumor in partial nephrectomy, Journal of Endourology Case Reports 4:1, 163-165, DOI: $10.1089 /$ cren.2018.0054. 